Kdp transport system, this ion at low concentrations stimulated and at high concentrations inhibited ATPase activity. Furthermore, Kdp ATPase activity was also inhibited by ADP, $N$-ethylmaleimide and vanadate, but not by ouabain or dicyclohexylcarbodi-imide.

Incubation of the Kdp ATPase with $\left[\gamma_{-32}\right.$ P]ATP revealed that in analogy to eukaryotic ion pumps a phosphorylated intermediate was formed during ATP hydrolysis. The. participation of this phosphoprotein in a phosphorylationdephosphorylation cycle was confirmed by pulse-chase experiments. Addition of non-radioactive ATP led to a fast turnover of the ${ }^{32} \mathrm{P}$-labelled intermediate. Both the $\mathrm{pH}$ lability profile of the intermediate and its sensitivity towards hydroxylamine suggest the involvement of an acylphosphate bond, which has tentatively be assigned to an aspartyl residue on subunit $B . K^{+}$induced a partial turnover of the phosphorylated intermediate; $\mathrm{Rb}^{+}, \mathrm{Na}^{+}$and $\mathrm{Li}^{+}$ were less effective. Both phosphorylation and dephosphorylation were strictly dependent upon $\mathrm{Mg}^{2+}$. Vanadate and $N$-ethylmaleimide blocked dephosphorylation.

Genetic analysis of $K_{M}$ mutants of the $K d p$ system suggested that the $k d p \mathrm{~A}$ gene product is involved in $\mathrm{K}^{+}$ binding; as yet direct evidence for its involvement in the $\mathrm{K}^{+}$-translocation step is lacking.

Biochemical studies have revealed that ATPase activity is associated with the $\mathrm{KdpB}$ subunit. The function of the $\mathrm{KdpC}$ protein still remains obscure. Since this subunit is absolutely necessary for transport activity it may play a role in the proper assembly of the enzyme complex (Epstein \& Davies, 1970).

Proteolytic digestion and radioactive-surface-labelling studies with diazobenzenesulphonic acid on right-side-out and everted vesicles indicated that the bulk of the $\mathrm{KdpB}$ protein is protruding from both sides of the membrane. By contrast, the main portions of the $\mathrm{KdpA}$ and $\mathrm{KdpC}$ proteins appear to be embedded in the membrane. Starting from the purified enzyme complex the three subunits of the Kdp ATPase have been isolated by sodium dodecyl sulphatecolumn chromatography. So far, the amino acid composition and the $N$-terminal sequence of subunit $C$ have been determined. Furthermore, the $k d p A B C$ operon has been cloned and the DNA sequences of the three subunits have partially been established.

Damper, P. D., Epstein, W., Rosen, B. P. \& Sorensen, E. N. (1979) Biochemistry 18, 4165-4169

Epstein, W. \& Davies, M. (1970) J. Bacteriol. 101, 836-843

Epstein, W., Whitelaw, V. \& Hesse, J. (1978) J. Biol. Chem. 253, 6666-6668

Laimins, L. A., Rhoads, D. B., Altendorf, K. \& Epstein, W. (1978) Proc. Natl. Acad. Sci. U.S.A. 75, 3216-3219

Laimins, L. A., Rhoads, D. B. \& Epstein, W. (1981) Proc. Natl. Acad. Sci. U.S.A. 78, 464-468

Rhoads, D. B. \& Epstein, W. (1978) J. Gen. Physiol. 72, 283295

Wieczorek, L. \& Altendorf, K. (1979) FEBS Lett. 98, 233-236

\title{
Isolation and manipulation of genes coding for energy-transducing enzymes from Neurospora crassa and Escherichia coli
}

\section{WALTER SEBALD, HERMANN ARENDS and JOHN E. G. MCCARTHY \\ Department of Cytogenetics, GBF-Gesellschaft für Biotechnologische Forschung m.b.H., Mascheroder Weg 1, D-3300 Braunschweig, Federal Republic of Germany}

Energy-transducing enzymes of the mitochondrial inner membrane, including ATP synthase, the $b c_{1}$ complex and cytochrome oxidase, have complicated subunit compositions. The cloning and isolation of the coding sequences (mRNAs or genes) will produce the possibility of gathering information on the amino acid sequences of these subunits. Moreover, the isolated nucleic acids can potentially be manipulated to allow study of the functional properties of these proteins. Some of these subunits are coded on mitochondrial DNA, and their corresponding genes have already been analysed and sequenced due to the relatively small size of this genome (Sloninsmiski et al., 1982). Most of the subunits are, however, encoded in the nucleus, ,translated in the cytosol (some of them as larger precursors) and subsequently imported into mitochondria. Owing to the large size of the nuclear genome, cloning and isolation of these genes is more difficult.

The ATP synthase occurs in remarkably similar form in mitochondria, chloroplasts and bacteria. Therefore the essential properties of the subunits can be studied by a comparison of the primary structure from phylogenetically distant organisms. In Escherichia coli all eight subunit genes are organized as one operon (the atp operon), and they have been cloned on $\lambda$ phages (Miki et al., 1978; Hansen \& von Meyenburg, 1980). In addition, mutants are available where single or several subunit genes are deleted. The $E$. coli system is therefore the system of choice for the expression of single subunits of the ATP synthase, and for the study of the resultant phenotypes (Friedl et al., 1983).

\section{Isolation of cloned mRNA from Neurospora crassa}

It can be estimated that in the lower eukaryote Neurospora crassa the mRNAs encoding subunits of mitochondrial energy-transducing complexes occur at frequencies of approx. 0.5-5 per 1000 (Viebrock et al., 1982). In order to identify a cloned mRNA of this abundancy, in an ordered cDNA bank, methods were adapted for the cell-free translation of hybrid-selected mRNA. The techniques were optimized so that in one experiment about 1000 clones could be analysed. Furthermore, the cell-free translation products of the hybrid-selected mRNAs could be precipitated with several specific antibodies, either simultaneously or in separate, successive incubations. Several rounds of hybridization could be performed, since cDNA plasmids had been covalently coupled to diazobenzyloxymethyl-paper.

Up to now, cDNAs have been identified which code for the proteolipid subunit and the $\delta$ subunit of the ATP synthase, for the ATP/ADP translocator, and for the ironsulphur protein and two of the smaller subunits of the $b c_{1}$ complex. cDNAs corresponding to most of each of the mRNAs of the proteolipid and of the ATP/ADP carrier have been sequenced, and the corresponding genes isolated and analysed.

\section{Characteristics of cloned proteins and genes from Neurospora}

The ATP synthase proteolipid subunit, an extremely hydrophobic protein of 81 amino acid residues, is synthesized as a precursor with a presequence of 66 amino acids. The presequence is very polar. It contains 12 basic residues and no acidic ones. The presequence apparently renders the proteolipid soluble, so that it can be imported into mitochondria post-translationally.

The nuclear gene contains two introns, one in the middle of the presequence, and one just before the last amino acid 
codon. The promotor region shows two homologous CTrich segments of 16 base-pairs. The mRNA shows two starts located at corresponding nucleotides in these segments.

The ATP/ADP translocator from Neurospora contains 313 amino acids, and is thus a few residues longer than the protein from beef heart mitochondria whose amino acid sequence has been recently determined by protein-chemical analysis (Aquila et al., 1982). The Neurospora carrier exhibits a high degree of homology to the beef heart protein. Interestingly, homology is restricted to six 25-35-residue segments which correspond to the hydrophobic domains of the polypeptide chain. The true start of the functional protein is not yet known but the primary translation product exhibits the same apparent $M_{\mathrm{r}}$ as the functional protein.

The gene contains two short introns after the 10th and 45 th amino acid codon. As with the proteolipid gene, the promoter regions show several CT-rich stretches. These stretches may be common to genes encoding middleabundant proteins such as the ATP/ADP translocator and the proteolipid.

Manipulation of genes of the proton-coducting $F_{0}$ part of ATP synthase

The $\mathrm{F}_{0}$ portion of the ATP synthase from E. coli contains three subunits. Although the mitochondrial $F_{0}$ appears to be more complex, two homologous proteins have been identified, i.e. the proteolipid and subunit 6. The atp operon, coding for all eight ATP-synthase subunits from $E$. coli, has been cloned on $\lambda$ phages by Hansen \& von Meyenburg (1980) and by Miki et al. (1978). The genes for the three $F_{0}$ subunits were cloned separately in order to study functional properties of individual subunits in vivo. Work is in progress to attach the individual genes to inducible promoters in order to achieve an in vivo reconstitution of $\mathrm{F}_{0}$ in whole cells.

Aquila, H., Misra, D., Eulitz, M. \& Klingenberg, M. (1982) Hoppe-Seyler's Z. Physiol. Chem. 363, 345-349

Friedl, P., Hoppe, J., Gunsalus, R. P., Michelsen, O., von Meyenburg, K. \& Schairer, H. U. (1983) EMBO J. 2, 99-103

Hansen, F. \& von Meyenburg, K. (1980) in Mechanistic Studies of DNA Replication and Genetic Recombination, (Alberts, B. \& Fox, C. F., eds.), vol. 19, pp. 137-159, ICN-UCLA Symposia on Molecular and Cellular Biology, Academic Press Inc. New York

Miki, T., Hiraga, S., Nagata, T. \& Yura, T. (1978) Proc. Natl. Acad. Sci. U.S.A. 75, 5099-5103

Sloninsmki, P., Borst, P. \& Attardi, G. (1982) Mitochondrial Genes, pp. 1-500, Cold Spring Harbor Monograph Series

Viebrock, A., Perz, A. \& Sebald, W. (1982) EMBO J. 1, 565571

\section{Molecular and genetic aspects of the fumarate reductase of Escherichia coli}

\section{STEWART T. COLE \\ Max-Planck-Institut für Biologie, Tübingen, Federal Republic of Germany}

Fumarate reductase is an anaerobically inducible, membrane-bound flavoprotein complex which effects the terminal step of an anaerobic electron-transport chain, employing fumarate as the ultimate electron acceptor. Electrons obtained from abundant substrates such as $\alpha$-glycerophosphate, lactate, formate, hydrogen or NADH are transferred via specific dehydrogenases, $b$-type cytochromes and menaquinone to fumarate reductase which then reduces fumarate to succinate. This process, common among both facultative and obligate anaerobic bacteria, generates a protonmotive force which can be used for the synthesis of ATP or for active transport (Kröger, 1978).

The best-understood fumarate reductase is that from Escherichia coli as it has been extensively characterized at both molecular genetic and biochemical levels. Initial studies with a specific group of mutants unable to grow anaerobically on non-fermentable substrates with fumarate as terminal electron acceptor identified the structural genes $(f r d)$ for the enzyme at $94 \mathrm{~min}$ on the $E$. coli linkage map and showed the reductase to be distinct from the tricarboxylic acid cycle enzyme, succinate dehydrogenase, which also interconverts fumarate and succinate (Hirsch et al., 1963; Spencer \& Guest, 1973; Bachmann \& Low, 1980). By means of complementation of defined frd lesions, specialized transducing phages bearing the genes encoding fumarate reductase were isolated from $\lambda$ gene banks (Cole \& Guest, $1978,1980 a$ ). These phages all contained a $4.9 \times 10^{3}$ basepair HindIII fragment which was later shown to be present in the Clarke and Carbon gene bank (Edlund et al., 1979; Guest, 1981). Determination of the entire nucleotide sequence of this fragment revealed the frd genes to be arranged in an operon (Fig. 1a) consisting of a regulatory region followed by four cistrons, frd $A, B, C, D$, coding for components of the enzyme, and a $\rho$-independent transcriptional terminator (Cole, 1982; Cole et al., 1982; Grundtröm \& Jaurin, 1982). A striking feature of the operon is its remarkable sequence economy: the frdA cistron partially overlaps fr $d B$ whereas the most distal cistron $f r d D$ contains the promoter region for the next gene, $a m p C$, within its coding sequence (Fig. 1a). One consequence of the latter arrangement is that the frd transcriptional terminator also serves to attenuate the growth-rate-dependent expression of the $a m p C$ gene (Grundström \& Jaurin, 1982). Further sequence economy is found within the intercistronic regions as in two cases the translational stop codons have been incorporated into the ribosome-binding sites of the following cistron.

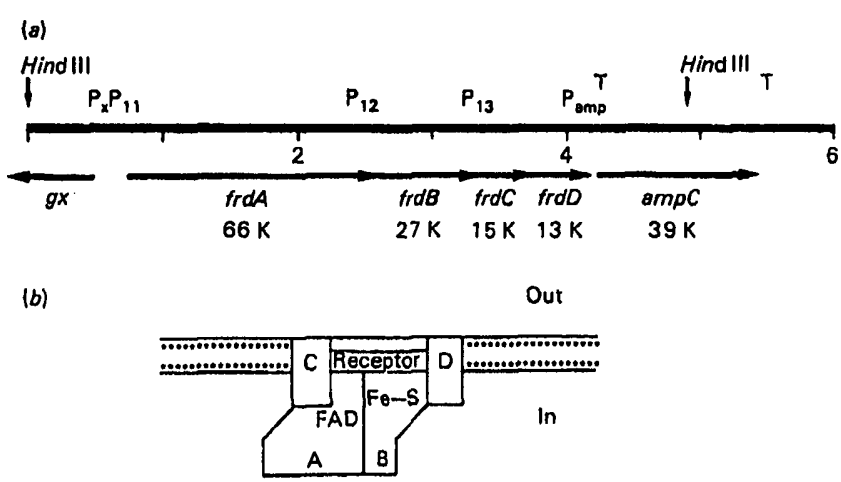

Fig. 1. Organization of the frd genes of $E$. coli and a model for the arrangement of fumarate reductase in the membrane

(a) The precise locations of the frdA, B,C,D and ampC genes, transcriptional terminators and the approximate location of the promoters are shown together with the $M_{\mathrm{r}}$ of the proteins they encode ( $K$, kilodaltons). An unidentified gene is denoted $g x$. The relevant restriction sites and the scale in $10^{3} \times$ base-pairs are indicated. (b) A schematic model for the arrangement of fumarate reductase in the membrane. Note that the precise association of the four subunits $(A, B, C, D)$ is not known. 